\title{
Reporting Score Distributions Makes a Difference: Performance Study of LSTM-networks for Sequence Tagging
}

\author{
Nils Reimers and Iryna Gurevych \\ Ubiquitous Knowledge Processing Lab (UKP) and Research Training Group AIPHES \\ Department of Computer Science, Technische Universität Darmstadt \\ Ubiquitous Knowledge Processing Lab (UKP-DIPF) \\ German Institute for Educational Research \\ www.ukp.tu-darmstadt.de
}

\begin{abstract}
In this paper we show that reporting a single performance score is insufficient to compare non-deterministic approaches. We demonstrate for common sequence tagging tasks that the seed value for the random number generator can result in statistically significant $\left(p<10^{-4}\right)$ differences for state-of-the-art systems. For two recent systems for NER, we observe an absolute difference of one percentage point $F_{1}$-score depending on the selected seed value, making these systems perceived either as state-of-the-art or mediocre. Instead of publishing and reporting single performance scores, we propose to compare score distributions based on multiple executions. Based on the evaluation of 50.000 LSTMnetworks for five sequence tagging tasks, we present network architectures that produce both superior performance as well as are more stable with respect to the remaining hyperparameters. The full experimental results are published in (Reimers and Gurevych, 2017). ${ }^{1}$ The implementation of our network is publicly available. ${ }^{2}$
\end{abstract}

\section{Introduction}

Large efforts are spent in our community on developing new state-of-the-art approaches. To document that those approaches are better, they are applied to unseen data and the obtained performance score is compared to previous approaches. In order to make results comparable, a provided split between train, development and test data is often

\footnotetext{
${ }^{1}$ https://arxiv.org/abs/1707.06799

${ }^{2}$ https: //github.com/UKPLab/ emnlp2017-bilstm-cnn-crf
}

used, for example from a former shared task.

In recent years, deep neural networks were shown to achieve state-of-the-art performance for a wide range of NLP tasks, including many sequence tagging tasks (Ma and Hovy, 2016), dependency parsing (Andor et al., 2016), and machine translation (Wu et al., 2016). The training process for neural networks is highly non-deterministic as it usually depends on a random weight initialization, a random shuffling of the training data for each epoch, and repeatedly applying random dropout masks. The error function of a neural network is a highly non-convex function of the parameters with the potential for many distinct local minima (LeCun et al., 1998; Erhan et al., 2010). Depending on the seed value for the pseudo-random number generator, the network will converge to a different local minimum.

Our experiments show that these different local minima have vastly different characteristics on unseen data. For the recent NER system by Ma and Hovy (2016) we observed that, depending on the random seed value, the performance on unseen data varies between $89.99 \%$ and $91.00 \% F_{1}$-score. The difference between the best and worst performance is statistically significant $\left(p<10^{-4}\right)$ using a randomization test ${ }^{3}$. In conclusion, whether this newly developed approach is perceived as state-of-the-art or as mediocre, largely depends on which random seed value is selected. This issue is not limited to this specific approach, but potentially applies to all approaches with non-deterministic training processes.

This large dependence on the random seed value creates several challenges when evaluating new approaches:

\footnotetext{
${ }^{3} 1$ Million iterations. p-value adapted using the Bonferroni correction to take the 86 tested seed values into account.
} 
- Observing a (statistically significant) improvement through a new non-deterministic approach might not be the result of a superior approach, but the result of having a more favorable sequence of random numbers.

- Promising approaches might be rejected too early, as they fail to deliver an outperformance simply due to a less favorable sequence of random numbers.

- Reproducing results is difficult.

To study the impact of the random seed value on the performance we will focus on five linguistic sequence tagging tasks: POS-tagging, Chunking, Named Entity Recognition, Entity Recognition $^{4}$, and Event Detection. Further we will focus on Long-Short-Term-Memory (LSTM) Networks (Hochreiter and Schmidhuber, 1997b), as those demonstrated state-of-the-art performance for a wide variety of sequence tagging tasks (Ma and Hovy, 2016; Lample et al., 2016; Søgaard and Goldberg, 2016).

Fixing the random seed value would solve the issue with the reproducibility, however, there is no justification for choosing one seed value over another seed value. Hence, instead of reporting and comparing a single performance, we show that comparing score distributions can lead to new insights into the functioning of algorithms.

Our main contributions are:

1. Showing the implications of non-deterministic approaches on the evaluation of approaches and the requirement to compare score distributions instead of single performance scores.

2. Comparison of two recent, state-of-the-art systems for NER and showing that reporting a single performance score can be misleading.

3. In-depth analysis of different LSTMarchitectures for five sequence tagging tasks with respect to: superior performance, stability of results, and importance of tuning parameters.

\footnotetext{
${ }^{4}$ Entity Recognition labels all tokens that refer to an entity in a sentence, also generic phrases like U.S. president.
}

\section{Background}

Validating and reproducing results is an important activity in science to manifest the correctness of previous conclusions and to gain new insights into the presented approaches. Fokkens et al. (2013) show that reproducing results is not always straightforward, as factors like preprocessing (e.g. tokenization), experimental setup (e.g. splitting data), the version of components, the exact implementation of features, and the treatment of ties can have a major impact on the achieved performance and sometimes on the drawn conclusions.

For approaches with non-deterministic training procedures, like neural networks, reproducing exact results becomes even more difficult, as randomness can play a major role in the outcome of experiments. The error function of a neural network is a highly non-convex function of the parameters with the potential for many distinct local minima (LeCun et al., 1998; Erhan et al., 2010). The sequence of random numbers plays a major role to which minima the network converges during the training process. However, not all minima generalize equally well to unseen data. Erhan et al. (2010) showed for the MNIST handwritten digit recognition task that different random seeds result in largely varying performances. They noted further that with increasing depth of the neural network, the probability of finding poor local minima increases.

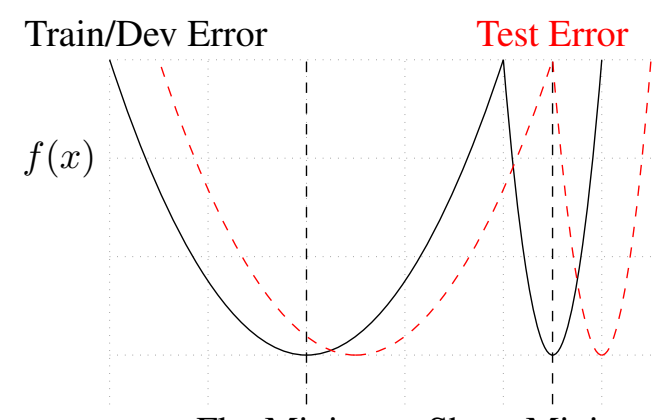

Flat Minimum Sharp Minimum

Figure 1: A conceptual sketch of flat and sharp minima from Keskar et al. (2016). The Y-axis indicates values of the error function and the $\mathrm{X}$ axis the weight-space.

As (informally) defined by Hochreiter and Schmidhuber (1997a), a minimum can be flat, where the error function remains approximately constant for a large connected region in weight-space, or it can be sharp, where the error function increases rapidly in 
a small neighborhood of the minimum. A conceptual sketch is given in Figure 1. The error functions for training and testing are typically not perfectly synced, i.e. the local minima on the train or development set are not the local minima for the held-out test set. A sharp minimum usually depicts poorer generalization capabilities, as a slight variation results in a rapid increase of the error function. On the other hand, flat minima generalize better on new data (Keskar et al., 2016). Keskar et al. observe for the MNIST, TIMIT, and CIFAR dataset, that the generalization gap is not due to over-fitting or over-training, but due to different generalization capabilities of the local minima the networks converge to.

A priori it is unknown to which type of local minimum a neural network will converge. Some methods like the weight initialization (Erhan et al., 2010; Glorot and Bengio, 2010) or small-batch training (Keskar et al., 2016) help to avoid bad (e.g. sharp) minima. Nonetheless, the non-deterministic behavior of approaches must be considered when they are evaluated.

\section{Impact of Randomness in the Evaluation of Neural Networks}

Two recent, state-of-the-art systems for NER are proposed by Ma and Hovy $(2016)^{5}$ and by Lample et al. $(2016)^{6}$. Lample et al. report an $F_{1}$-score of $90.94 \%$ and Ma and Hovy report an $F_{1}$-score of 91.21\%. Ma and Hovy draw the conclusion that their system achieves a significant improvement over the system by Lample et al.

We re-ran both implementations multiple times, each time only changing the seed value of the random number generator. We ran the Ma and Hovy system 86 times and the Lample et al. system, due to its high computational requirement, for 41 times. The score distribution is depicted as a violin plot in Figure 2. Using a Kolmogorov-Smirnov significance test (Massey, 1951), we observe a statistically significant difference between these two distributions $(p<0.01)$. The plot reveals that the quartiles for the Lample et al. system are above those of the Ma and Hovy system. Further it reveals a smaller standard deviation $\sigma$ of the $F_{1}$ -

\footnotetext{
${ }^{5}$ https://github.com/XuezheMax/ LasagneNLP

${ }^{6}$ https://github.com/glample/tagger
}

scores for the Lample et al. system. Using a BrownForsythe test, the standard deviations are different with $p<0.05$. Table 1 shows the minimum, the maximum, and the median performance for the test performances.

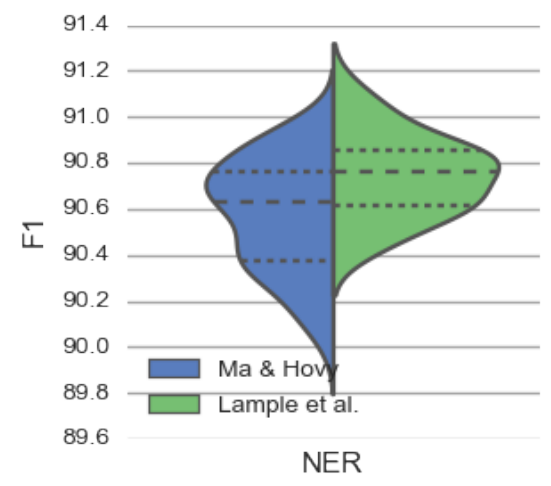

Figure 2: Distribution of scores for re-running the system by Ma and Hovy (left) and Lample et al. (right) multiple times with different seed values. Dashed lines indicate quartiles.

Based on this observation, we draw the conclusion that the system by Lample et al. outperforms the system by Ma and Hovy, as their implementation achieves a higher score distribution and shows a lower standard deviation.

In a usual setup, approaches would be compared on a development set and the run with the highest development score would be used for unseen data, i.e. be used to report the test performance. For the Lample et al. system we observe a Spearman's rank correlation between the development and the test score of $\rho=0.229$. This indicates a weak correlation and that the performance on the development set is not a reliable indicator. Using the run with the best development score (94.44\%) would yield a test performance of mere $90.31 \%$. Using the second best run on development set (94.28\%), would yield state-of-the-art performance with $91.00 \%$. This difference is statistically significant $(p<0.002)$. In conclusion, a development set will not necessarily solve the issue with bad local minima.

The main difference between these two approaches is in the generation of character-based representations: Ma and Hovy uses a Convolutional Neural Network (CNN) (LeCun et al., 1989), while Lample et al. uses an LSTM-network. As our experiments in section 6.4 show, both approaches perform comparably if all other parameters were kept the same. Further, we could only observe a 


\begin{tabular}{|c|c||c|c|c|c|c|}
\hline System & Reported $F_{1}$ & \# Seed values & Min. $F_{1}$ & Median $F_{1}$ & Max. $F_{1}$ & $\sigma$ \\
\hline Ma and Hovy & $91.21 \%$ & 86 & $89.99 \%$ & $90.64 \%$ & $91.00 \%$ & 0.00241 \\
\hline Lample et al. & $90.94 \%$ & 41 & $90.19 \%$ & $90.81 \%$ & $91.14 \%$ & 0.00176 \\
\hline
\end{tabular}

Table 1: The system by Ma and Hovy (2016) and Lample et al. (2016) were run multiple times with different seed values.

\begin{tabular}{|c|c|c|c|c|c|}
\hline Task & Dataset & \# Configs & Median Difference & 95th percentile & Max. Difference \\
\hline POS & Penn Treebank & 269 & $0.17 \%$ & $0.78 \%$ & $1.55 \%$ \\
\hline Chunking & CoNLL 2000 & 385 & $0.17 \%$ & $0.50 \%$ & $0.81 \%$ \\
\hline NER & CoNLL 2003 & 406 & $0.38 \%$ & $1.08 \%$ & $2.59 \%$ \\
\hline Entities & ACE 2005 & 405 & $0.72 \%$ & $2.10 \%$ & $8.23 \%$ \\
\hline Events & TempEval 3 & 365 & $0.43 \%$ & $1.23 \%$ & $1.73 \%$ \\
\hline
\end{tabular}

Table 2: The table depicts the median, the 95th percentile and the maximum difference between networks with the same hyperparameters but different random seed values.

statistically significant improvement for the tasks POS, Chunking and Event Detection. For NER and Entity Recognition, the difference was statistically not significant given the number of tested hyperparameters.

In the next step, we evaluated the impact of the random seed value for the five sequence tagging tasks described in section 4. We sampled randomly 1830 different configurations, for example different numbers of recurrent units, and ran the network twice, each time with a different seed value. The results are depicted in Table 2.

The largest difference was observed for the ACE 2005 Entities dataset: Using one seed value, the network achieved an $F_{1}$ performance of $82.5 \%$ while using another seed value, the network achieved a performance of only $74.3 \%$. Even though this is a rare extreme case, the median difference between different weight initializations is still large. For example for the CoNLL 2003 NER dataset, the median difference is at $0.38 \%$ and the 95 th percentile is at $1.08 \%$.

In conclusion, if the fact of different local minima is not taken care of and single performance scores are compared, there is a high chance of drawing false conclusions and either rejecting promising approaches or selecting weaker approaches due to a more or less favorable sequence of random numbers.

\section{Experimental Setup}

In order to find LSTM-network architectures that perform robustly on different tasks, we selected five classical NLP tasks as benchmark tasks: Partof-Speech tagging (POS), Chunking, Named Entity Recognition (NER), Entity Recognition (Entities) and Event Detection (Events).

For Part-of-Speech tagging, we use the benchmark setup described by Toutanova et al. (2003). Using the full training set for POS tagging would hinder our ability to detect design choices that are consistently better than others. The error rate for this dataset is approximately 3\% (Marcus et al., 1993), making all improvements above $97 \%$ accuracy likely the result of chance. A $97.24 \%$ accuracy was achieved by Toutanova et al. (2003). Hence, we reduced the training set size from over 38.000 sentences to the first 500 sentences. This decreased the accuracy to about $95 \%$.

For Chunking, we use the CoNLL 2000 shared task setup. For Named Entity Recognition (NER), we use the CoNLL 2003 setup. The ACE 2005 entity recognition task annotated not only named entities, but all words referring to an entity, e.g. the phrase U.S. president. We use the same data split as $\mathrm{Li}$ et al. (2013). For the Event Detection task, we use the TempEval3 Task B setup. There, the smallest extent of text, usually a single word, that expresses the occurrence of an event, is annotated.

For the POS-task, we report accuracy and for the other tasks we report the $F_{1}$-score. 


\subsection{Model}

We use a BiLSTM-network for sequence tagging as described in (Huang et al., 2015; Ma and Hovy, 2016; Lample et al., 2016). To be able to evaluate a large number of different network configurations, we optimized our implementation for efficiency, reducing by a factor of 6 the time required per epoch compared to Ma and Hovy (2016).

\subsection{Evaluated Parameters}

We evaluate the following design choices and hyperparameters:

Pre-trained Word Embeddings. We evaluate the Google News embeddings $(\text { G. News })^{7}$ from Mikolov et al. (2013), the Bag of Words (Le $B o W)$ as well as the dependency based embeddings (Le. Dep. $)^{8}$ by Levy and Goldberg (2014), three different GloVe embeddings ${ }^{9}$ from Pennington et al. (2014) trained either on Wikipedia 2014 + Gigaword 5 (GloVe1 with 100 dimensions and GloVe2 with 300 dimensions) or on Common Crawl (GloVe3), and the Komninos and Manandhar (2016) embeddings (Komn. $)^{10}$. We also evaluate the approach of Bojanowski et al. (2016) (Fast$T e x t$ ), which trains embeddings for n-grams with length 3 to 6 . The embedding for a word is defined as the sum of the embeddings of the ngrams.

Character Representation. We evaluate the approaches of Ma and Hovy (2016) using Convolutional Neural Networks (CNN) as well as the approach of Lample et al. (2016) using LSTMnetworks to derive character-based representations.

Optimizer. Besides Stochastic Gradient Descent (SGD), we evaluate Adagrad (Duchi et al., 2011), Adadelta (Zeiler, 2012), RMSProp (Hinton, 2012), Adam (Kingma and Ba, 2014), and Nadam (Dozat, 2015), an Adam variant that incorporates Nesterov momentum (Nesterov, 1983) as optimizers.

Gradient Clipping and Normalization. Two common strategies to deal with the exploding gradi-

\footnotetext{
${ }^{7}$ https://code.google.com/archive/p/ word2vec/

${ }^{8}$ https: / / levyomer.wordpress.com/2014/ $04 / 25$ / dependency-based-word-embeddings /

${ }^{9}$ http://nlp.stanford.edu/projects/ glove/

${ }^{10}$ https://www.cs.york.ac.uk/nlp/extvec/
}

ent problem are gradient clipping (Mikolov, 2012) and gradient normalization (Pascanu et al., 2013). Gradient clipping involves clipping the gradient's components element-wise if it exceeds a defined threshold. Gradient normalization has a better theoretical justification and rescales the gradient whenever the norm goes over a threshold.

Tagging schemes. We evaluate the $B I O$ and IOBES schemes for tagging segments.

Dropout. We compare no dropout, naive dropout, and variational dropout (Gal and Ghahramani, 2016). Naive dropout applies a new dropout mask at every time step of the LSTM-layers. Variational dropout applies the same dropout mask for all time steps in the same sentence. Further, it applies dropout to the recurrent units. We evaluate the dropout rates $\{0.05,0.1,0.25,0.5\}$.

Classifier. We evaluate a Softmax classifier as well as a CRF classifier as the last layer of the network.

Number of LSTM-layers. We evaluated 1, 2, and 3 stacked BiLSTM-layers.

Number of recurrent units. For each LSTM-layer, we selected independently a number of recurrent units from the set $\{25,50,75,100,125\}$.

Mini-batch sizes. We evaluate the mini-batch sizes $1,8,16,32$, and 64 .

\section{Robust Model Evaluation}

We have shown in section 3 that re-running nondeterministic approaches multiple times and comparing score distributions is essential to draw correct conclusions. However, to truly understand the capabilities of an approach, it is interesting to test the approach with different sets of hyperparameters for the complete network.

Training and tuning a neural network can be time consuming, sometimes taking multiple days to train a single instance of a network. A priori it is hard to know which hyperparameters will yield the best performance and the selection of the parameters often makes the difference between mediocre and state-of-the-art performance (Hutter et al., 2014). If an approach yields good performance only for a narrow set of parameters, it might be difficult to adapt the approach to new tasks, new domains 
or new languages, as a large range of possible parameters must be evaluated, each time requiring a significant amount of training time. Hence it is desirable, that the approach yields stable results for a wide range of parameters.

In order to find approaches that result in high performance and are robust against the remaining parameters, we decided to randomly sample several hundred network configurations from the set described in section 4.2. For each sampled configuration, we compare different options, e.g. different options for the last layer of the network. For example, we sampled in total 975 configurations and each configuration was trained with a Softmax classifier as well as with a CRF classifier, totaling to 1950 trained networks.

\begin{tabular}{|c|c|c|c|}
\hline Dataset & \# Configs & Softmax & CRF \\
\hline POS & 111 & $18.9 \%$ & $\mathbf{8 1 . 1 \%}$ \\
$\Delta$ Acc. & & $-0.20 \%$ & \\
\hline Chunking & 229 & $4.8 \%$ & $\mathbf{9 5 . 2 \%}$ \\
$\Delta F_{1}$ & & $-0.38 \%$ & \\
\hline NER & 232 & $9.5 \%$ & $\mathbf{9 0 . 5 \%}$ \\
$\Delta F_{1}$ & & $-0.66 \%$ & \\
\hline Entities & 210 & $13.3 \%$ & $\mathbf{8 6 . 7 \%}$ \\
$\Delta F_{1}$ & & $-0.84 \%$ & \\
\hline Events & 202 & $\mathbf{6 1 . 9 \%}$ & $38.1 \%$ \\
$\Delta F_{1}$ & & & $-0.15 \%$ \\
\hline \hline Average & & $21.7 \%$ & $\mathbf{7 8 . 3 \%}$ \\
\hline
\end{tabular}

Table 3: Percentages of configurations where Softmax or CRF classifiers demonstrated a higher test performance.

Our results are presented in Table 3. The table shows that for the NER task 232 configurations were sampled randomly and for 210 of the 232 configurations (90.5\%), the CRF setup achieved a better test performance than the setup with a Softmax classifier. To measure the difference between these two options, we compute the median of the absolute differences: Let $S_{i}$ be the test performance ( $F_{1}$-measure) for the Softmax setup for configuration $i$ and $C_{i}$ the test performance for the CRF setup. We then compute $\Delta F_{1}=\operatorname{median}\left(S_{1}-\right.$ $\left.C_{1}, S_{2}-C_{2}, \ldots, S_{232}-C_{232}\right)$. For the NER task, the median difference was $\Delta F_{1}=-0.66 \%$, i.e. the setup with a Softmax classifier achieved on average an $F_{1}$-score of 0.66 percentage points below that of the CRF setup.

We also evaluated the standard deviation of the $F_{1^{-}}$ scores to detect approaches that are less dependent on the remaining hyperparameters and the random number generator. The standard deviation $\sigma$ for the CRF-classifier is with 0.0060 significantly lower ( $p<10^{-3}$ using Brown-Forsythe test) than for the Softmax classifier with $\sigma=0.0082$.

\section{Results}

This section highlights our main insights in the evaluation of different design choices for BiLSTM architectures. We limit the number of results we present for reasons of brevity. Detailed information can be found in (Reimers and Gurevych, 2017). ${ }^{11}$

\subsection{Classifier}

Table 3 shows a comparison between using a Softmax classifier as a last layer and using a CRF classifier. The BiLSTM-CRF architecture by Huang et al. (2015) achieves a better performance on 4 out of 5 tasks. For the NER task it further achieves a $27 \%$ lower standard deviation (statistically significant with $p<10^{-3}$ ), indicating that it is less sensitive to the remaining configuration of the network.

The CRF classifier only fails for the Event Detection task. This task has nearly no dependency between tags, as often only a single token is annotated as an event trigger in a sentence.

We studied the differences between these two classifiers in terms of number of LSTM-layers. As Figure 3 shows, a Softmax classifier profits from a deep LSTM-network with multiple stacked layers. On the other hand, if a CRF classifier is used, the effect of additional LSTM-layers is much smaller.

\subsection{Optimizer}

We evaluated six optimizers with the suggested default configuration from their respective papers. We observed that SGD is quite sensitive towards the selection of the learning rate and it failed in many instances to converge. For the optimizers SGD, Adagrad and Adadelta we observed a large standard deviation in terms of test performance,

\footnotetext{
${ }^{11}$ https://public.ukp. informatik. tu-darmstadt.de/reimers/optimal_ Hyperparameters_for_Deep_LSTM-Networks. pdf
} 


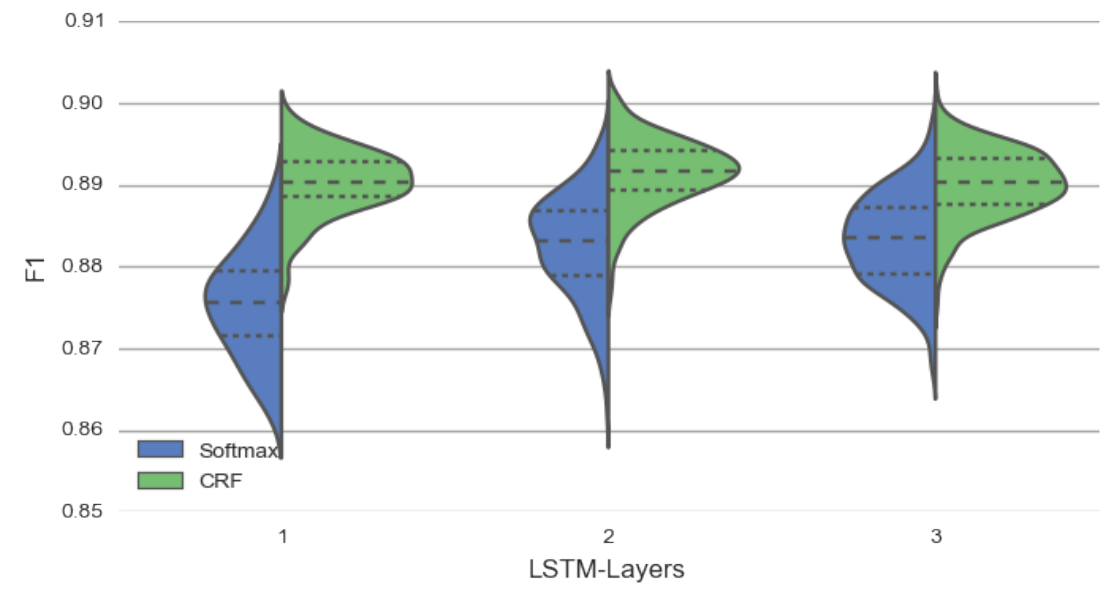

Figure 3: Difference between Softmax and CRF classifier for different number of BiLSTM-layers for the CoNLL 2003 NER dataset.

which was for the NER task at 0.1328 for SGD, 0.0139 for Adagrad, and 0.0138 for Adadelta. The optimizers RMSProp, Adam, and Nadam on the other hand produced much more stable results. Not only were the medians for these three optimizers higher, but also the standard deviation was with $0.0096,0.0091$, and 0.0092 roughly $35 \%$ smaller in comparison to Adagrad. A large standard deviation indicates that the optimizer is sensitive to the hyperparameters as well as to the random initialization and bears the risk that the optimizer produces subpar results.

The best result was achieved by Nadam. For 453 out of 882 configurations (51.4\%), it yielded the highest performance out of the six tested optimizers. For the NER task, it produced on average a 0.82 percentage points better performance than Adagrad.

Besides test performance, the convergence speed is important in order to reduce training time. Here, Nadam had the best convergence speed. For the NER dataset, Nadam converged on average after 9 epochs, whereas SGD required 42 epochs.

\subsection{Word Embeddings}

The pre-trained word embeddings had a large impact on the performance as shown in Table 4 . The embeddings by Komninos and Manandhar (2016) resulted in the best performance for the POS, the Entities and the Events task. For the Chunking task, the dependency-based embeddings of Levy and Goldberg (2014) are slightly ahead of the Komninos embeddings, the significance level is at $p=0.025$. For NER, the GloVe embeddings trained on common crawl perform on par with the Komninos embeddings $(p=0.391)$.

We observe that the underlying word embeddings have a large impact on the performance for all tasks. Well suited word embeddings are especially critical for datasets with small training sets. For the POS task we observe a median difference of $4.97 \%$ between the Komninos embeddings and the GloVe2 embeddings.

Note we only evaluated the pre-trained embeddings provided by different authors, but not the underlying algorithms to generate these embeddings. The quality of word embeddings depends on many factors, including the size, the quality, and the preprocessing of the data corpus. As the corpora are not comparable, our results do not allow concluding that one approach is superior for generating word embeddings.

\subsection{Character Representation}

We evaluate the approaches of Ma and Hovy (2016) using Convolutional Neural Networks (CNN) as well as the approach of Lample et al. (2016) using LSTM-networks to derive character-based representations.

Table 5 shows that character-based representations yield a statistically significant difference only for the POS, the Chunking, and the Events task. For NER and Entity Recognition, the difference to not using a character-based representation is not significant $(p>0.01)$. 


\begin{tabular}{|c|c|c|c|c|c|c|c|c|}
\hline Dataset & Le. Dep. & Le. BoW & GloVe1 & GloVe2 & GloVe3 & Komn. & G. News & FastText \\
\hline POS & $6.5 \%$ & $0.0 \%$ & $0.0 \%$ & $0.0 \%$ & $0.0 \%$ & $\mathbf{9 3 . 5 \%}$ & $0.0 \%$ & $0.0 \%$ \\
$\Delta A c c$. & $-0.39 \%$ & $-2.52 \%$ & $-4.14 \%$ & $-4.97 \%$ & $-2.60 \%$ & & $-1.95 \%$ & $-2.28 \%$ \\
\hline Chunking & $\mathbf{6 0 . 8 \%}$ & $0.0 \%$ & $0.0 \%$ & $0.0 \%$ & $0.0 \%$ & $37.1 \%$ & $2.1 \%$ & $0.0 \%$ \\
$\Delta F_{1}$ & & $-0.52 \%$ & $-1.09 \%$ & $-1.50 \%$ & $-0.93 \%$ & $-0.10 \%$ & $-0.48 \%$ & $-0.75 \%$ \\
\hline NER & $4.5 \%$ & $0.0 \%$ & $22.7 \%$ & $0.0 \%$ & $\mathbf{4 3 . 6 \%}$ & $27.3 \%$ & $1.8 \%$ & $0.0 \%$ \\
$\Delta F_{1}$ & $-0.85 \%$ & $-1.17 \%$ & $-0.15 \%$ & $-0.73 \%$ & & $-0.08 \%$ & $-0.75 \%$ & $-0.89 \%$ \\
\hline Entities & $4.2 \%$ & $7.6 \%$ & $0.8 \%$ & $0.0 \%$ & $6.7 \%$ & $\mathbf{5 7 . 1 \%}$ & $21.8 \%$ & $1.7 \%$ \\
$\Delta F_{1}$ & $-0.92 \%$ & $-0.89 \%$ & $-1.50 \%$ & $-2.24 \%$ & $-0.80 \%$ & & $-0.33 \%$ & $-1.13 \%$ \\
\hline Events & $12.9 \%$ & $4.8 \%$ & $0.0 \%$ & $0.0 \%$ & $0.0 \%$ & $\mathbf{7 1 . 8 \%}$ & $9.7 \%$ & $0.8 \%$ \\
$\Delta F_{1}$ & $-0.55 \%$ & $-0.78 \%$ & $-2.77 \%$ & $-3.55 \%$ & $-2.55 \%$ & & $-0.67 \%$ & $-1.36 \%$ \\
\hline \hline Average & $17.8 \%$ & $2.5 \%$ & $4.7 \%$ & $0.0 \%$ & $10.1 \%$ & $\mathbf{5 7 . 4 \%}$ & $7.1 \%$ & $0.5 \%$ \\
\hline
\end{tabular}

Table 4: Randomly sampled configurations were evaluated with 8 possible word embeddings. 108 configurations were sampled for POS, 97 for Chunking, 110 for NER, 119 for Entities, and 124 for Events.

The difference between the CNN approach by Ma and Hovy (2016) and the LSTM approach by Lample et al. (2016) to derive a character-based representations is statistically insignificant for all tasks. This is quite surprising, as both approaches have fundamentally different properties: The CNN approach from Ma and Hovy (2016) takes only trigrams into account. It is also position independent, i.e. the network will not be able to distinguish between trigrams at the beginning, in the middle, or at the end of a word, which can be crucial information for some tasks. The BiLSTM approach from Lample et al. (2016) takes all characters of the word into account. Further, it is position aware, i.e. it can distinguish between characters at the start and at the end of the word. Intuitively, one would think that the LSTM approach by Lample et al. would be superior.

\subsection{Gradient Clipping and Normalization}

For gradient clipping (Mikolov, 2012) we couldn't observe any improvement for the thresholds of 1 , 3,5 , and 10 for any of the five tasks.

Gradient normalization has a better theoretical justification (Pascanu et al., 2013) and we can confirm with our experiments that it performs better. Not normalizing the gradient was the best option only for $5.6 \%$ of the 492 evaluated configurations (under null-hypothesis we would expect $20 \%$ ). Which threshold to choose, as long as it is not too small or too large, is of lower importance. In most cases, a threshold of 1 was the best option (30.5\% of the

\begin{tabular}{|c|c|c|c|}
\hline Task & No & CNN & LSTM \\
\hline POS & $4.9 \%$ & $\mathbf{5 8 . 2 \%}$ & $36.9 \%$ \\
$\Delta$ Acc. & $-0.90 \%$ & & $-0.05 \%$ \\
\hline Chunking & $13.3 \%$ & $43.2 \%$ & $\mathbf{4 3 . 6 \%}$ \\
$\Delta F_{1}$ & $-0.20 \%$ & $-0.00 \%$ & \\
\hline NER & $27.2 \%$ & $\mathbf{3 6 . 4 \%}$ & $36.4 \%$ \\
$\Delta F_{1}$ & $-0.11 \%$ & & $-0.01 \%$ \\
\hline Entities & $26.8 \%$ & $36.0 \%$ & $\mathbf{3 7 . 3 \%}$ \\
$\Delta F_{1}$ & $-0.07 \%$ & $0.00 \%$ & \\
\hline Events & $20.5 \%$ & $35.6 \%$ & $\mathbf{4 3 . 8 \%}$ \\
$\Delta F_{1}$ & $-0.44 \%$ & $-0.04 \%$ & \\
\hline Average & $18.5 \%$ & $\mathbf{4 1 . 9 \%}$ & $39.6 \%$ \\
\hline
\end{tabular}

Table 5: Comparison of not using character-based representations and using CNNs (Ma and Hovy, 2016) or LSTMs (Lample et al., 2016) to derive character-based representations. 225 configurations were sampled for POS, 241 for Chunking, 217 for NER, 228 for Entities, and 219 for Events.

cases).

We observed a large performance increase compared to not normalizing the gradient. The median increase was between 0.29 percentage points $F_{1}$ score for the Chunking task and 0.82 percentage points for the POS task.

\subsection{Dropout}

Dropout is a popular method to deal with overfitting for neural networks (Srivastava et al., 2014). We could observe that variational dropout (Gal and Ghahramani, 2016) clearly outperforms naive dropout and not using dropout. It was the best op- 
tion in $83.5 \%$ of the 479 evaluated configurations. The median performance increase in comparison to not using dropout was between 0.31 percentage points for the POS-task and 1.98 for the Entities task. We also observed a large improvement in comparison to naive dropout between 0.19 percentage points for the POS task and 1.32 percentage points for the Entities task. Variational dropout showed the smallest standard deviation, indicating that it is less dependent on the remaining hyperparameters and the random number sequence.

We further evaluated whether variational dropout should be applied to the output units of the LSTMnetwork, to the recurrent units, or to both. We observed that applying dropout to both dimensions gave in most cases (62.6\%) the best results. The median performance increase was between 0.05 percentage points and 0.82 percentage points.

\subsection{Further Evaluated Parameters}

The tagging schemes BIO and IOBES performed on par for 4 out of 5 tasks. For the Entities task, the BIO scheme significantly outperformed the IOBES scheme for $88.7 \%$ of the tested configurations. The median difference was $\Delta F_{1}=$ $-1.01 \%$.

For the evaluated tasks, 2 stacked LSTM-layers achieved the best performance. For the POStagging task, 1 and 2 layers performed on par. For flat networks with a single LSTM-layer, around 150 recurrent units yielded the best performance. For networks with 2 or 3 layers, around 100 recurrent units per network yielded the best performance. However, the impact of the number of recurrent units was extremely small.

For tasks with small training sets, smaller minibatch sizes of 1 up to 16 appears to be a good choice. For larger training sets sizes of $8-32$ appears to be a good choice. Mini-batch sizes of 64 usually performed worst.

\section{Conclusion}

In this paper, we demonstrated that the sequence of random numbers has a statistically significant impact on the test performance and that wrong conclusions can be made if performance scores based on single runs are compared. We demonstrated this for the two recent state-of-the-art NER systems by Ma and Hovy (2016) and Lample et al. (2016). Based on the published performance scores, Ma and Hovy draw the conclusion of a significant improvement over the approach of Lample et al. Re-executing the provided implementations with different seed values however showed that the implementation of Lample et al. results in a superior score distribution generalizing better to unseen data.

Comparing score distributions reduces the risk of rejecting promising approaches or falsely accepting weaker approaches. Further it can lead to new insights on the properties of an approach. We demonstrated this for ten design choices and hyperparameters of LSTM-networks for five tasks.

By studying the standard deviation of scores, we estimated the dependence on hyperparameters and on the random seed value for different approaches. We showed that SGD, Adagrad and Adadelta have a higher dependence than RMSProp, Adam or Nadam. We have shown that variational dropout also reduces the dependence on the hyperparameters and on the random seed value. As future work, we will investigate if those methods are either less dependent on the hyperparameters or are less dependent on the random seed value, e.g. if they avoid converging to bad local minima.

By testing a large number of configurations, we showed that some choices consistently lead to superior performance and are less dependent on the remaining configuration of the network. Thus, there is a good chance that these configurations require less tuning when applied to new tasks or domains.

\section{Acknowledgements}

This work has been supported by the German Research Foundation as part of the Research Training Group Adaptive Preparation of Information from Heterogeneous Sources (AIPHES) under grant No. GRK 1994/1. Calculations for this research were conducted on the Lichtenberg high performance computer of the TU Darmstadt. 


\section{References}

Daniel Andor, Chris Alberti, David Weiss, Aliaksei Severyn, Alessandro Presta, Kuzman Ganchev, Slav Petrov, and Michael Collins. 2016. Globally normalized transition-based neural networks. CoRR, abs/1603.06042.

Piotr Bojanowski, Edouard Grave, Armand Joulin, and Tomas Mikolov. 2016. Enriching Word Vectors with Subword Information. arXiv preprint arXiv:1607.04606.

Timothy Dozat. 2015. Incorporating Nesterov Momentum into Adam.

John Duchi, Elad Hazan, and Yoram Singer. 2011. Adaptive Subgradient Methods for Online Learning and Stochastic Optimization. J. Mach. Learn. Res., $12: 2121-2159$.

Dumitru Erhan, Yoshua Bengio, Aaron Courville, Pierre-Antoine Manzagol, Pascal Vincent, and Samy Bengio. 2010. Why Does Unsupervised Pre-training Help Deep Learning? Journal of Machine Learning Research, 11:625-660.

Antske Fokkens, Marieke van Erp, Marten Postma, Ted Pedersen, Piek Vossen, and Nuno Freire. 2013. Offspring from Reproduction Problems: What Replication Failure Teaches Us. In ACL (1), pages 1691-1701. The Association for Computer Linguistics.

Yarin Gal and Zoubin Ghahramani. 2016. A Theoretically Grounded Application of Dropout in Recurrent Neural Networks. In Advances in Neural Information Processing Systems 29: Annual Conference on Neural Information Processing Systems 2016, December 5-10, 2016, Barcelona, Spain, pages 1019-1027.

Xavier Glorot and Yoshua Bengio. 2010. Understanding the difficulty of training deep feedforward neural networks. In In Proceedings of the International Conference on Artificial Intelligence and Statistics (AISTATS10). Society for Artificial Intelligence and Statistics.

Geoffrey Hinton. 2012. Neural Networks for Machine Learning - Lecture $6 a$ - Overview of mini-batch gradient descent.

Sepp Hochreiter and Jürgen Schmidhuber. 1997a. Flat Minima. Neural Computation, 9(1):1-42.

Sepp Hochreiter and Jürgen Schmidhuber. 1997b. Long Short-Term Memory. Neural Computation, 9(8):1735-1780.

Zhiheng Huang, Wei Xu, and Kai Yu. 2015. Bidirectional LSTM-CRF Models for Sequence Tagging. CoRR, abs/1508.01991.

Frank Hutter, Holger Hoos, and Kevin Leyton-Brown. 2014. An Efficient Approach for Assessing Hyperparameter Importance. In Proceedings of the 31st International Conference on International Conference on Machine Learning - Volume 32, ICML'14, pages I754-I-762. JMLR.org.
Nitish Shirish Keskar, Dheevatsa Mudigere, Jorge Nocedal, Mikhail Smelyanskiy, and Ping Tak Peter Tang. 2016. On Large-Batch Training for Deep Learning: Generalization Gap and Sharp Minima. CoRR, abs/1609.04836.

Diederik P. Kingma and Jimmy Ba. 2014. Adam: A Method for Stochastic Optimization. CoRR, abs/1412.6980.

Alexandros Komninos and Suresh Manandhar. 2016. Dependency based embeddings for sentence classification tasks. In Proceedings of the 2016 Conference of the North American Chapter of the Association for Computational Linguistics: Human Language Technologies, pages 1490-1500, San Diego, California. Association for Computational Linguistics.

Guillaume Lample, Miguel Ballesteros, Sandeep Subramanian, Kazuya Kawakami, and Chris Dyer. 2016. Neural architectures for named entity recognition. CoRR, abs/1603.01360.

Y. LeCun, B. Boser, J. S. Denker, D. Henderson, R. E. Howard, W. Hubbard, and L. D. Jackel. 1989. Backpropagation Applied to Handwritten Zip Code Recognition. Neural Computation, 1(4):541-551.

Yann LeCun, Léon Bottou, Genevieve B. Orr, and Klaus-Robert Müller. 1998. Efficient BackProp. In Neural Networks: Tricks of the Trade, This Book is an Outgrowth of a 1996 NIPS Workshop, pages 9-50, London, UK, UK. Springer-Verlag.

Omer Levy and Yoav Goldberg. 2014. DependencyBased Word Embeddings. In Proceedings of the 52nd Annual Meeting of the Association for Computational Linguistics, ACL 2014, June 22-27, 2014, Baltimore, MD, USA, Volume 2: Short Papers, pages 302-308.

Qi Li, Heng Ji, and Liang Huang. 2013. Joint Event Extraction via Structured Prediction with Global Features. In Proceedings of the 51st Annual Meeting of the Association for Computational Linguistics (Volume 1: Long Papers), pages 73-82, Sofia, Bulgaria. Association for Computational Linguistics.

Xuezhe Ma and Eduard H. Hovy. 2016. End-to-end Sequence Labeling via Bi-directional LSTM-CNNs-CRF. CoRR, abs/1603.01354.

Mitchell P. Marcus, Mary Ann Marcinkiewicz, and Beatrice Santorini. 1993. Building a Large Annotated Corpus of English: The Penn Treebank. Comput. Linguist., 19(2):313-330.

Frank J. Massey. 1951. The kolmogorov-smirnov test for goodness of fit. Journal of the American Statistical Association, 46(253):68-78.

Tomáš Mikolov. 2012. Statistical language models based on neural networks. Ph.D. thesis, Brno University of Technology.

Tomas Mikolov, Kai Chen, Greg Corrado, and Jeffrey Dean. 2013. Efficient Estimation of Word Representations in Vector Space. CoRR, abs/1301.3781. 
Yurii Nesterov. 1983. A method of solving a convex programming problem with convergence rate $\mathrm{O}(1 / \mathrm{sqr}(\mathrm{k}))$. Soviet Mathematics Doklady, 27:372376.

Razvan Pascanu, Tomas Mikolov, and Yoshua Bengio. 2013. On the Difficulty of Training Recurrent Neural Networks. In Proceedings of the 30th International Conference on International Conference on Machine Learning - Volume 28, ICML'13, pages III-1310-III1318. JMLR.org.

Jeffrey Pennington, Richard Socher, and Christopher D. Manning. 2014. Glove: Global vectors for word representation. In Empirical Methods in Natural Language Processing (EMNLP), pages 1532-1543.

Nils Reimers and Iryna Gurevych. 2017. Optimal Hyperparameters for Deep LSTM-Networks for Sequence Labeling Tasks. arXiv preprint arXiv:1707.06799.

Anders Søgaard and Yoav Goldberg. 2016. Deep multitask learning with low level tasks supervised at lower layers. In Proceedings of the 54th Annual Meeting of the Association for Computational Linguistics (Volume 2: Short Papers), pages 231-235, Berlin, Germany. Association for Computational Linguistics.

Nitish Srivastava, Geoffrey Hinton, Alex Krizhevsky, Ilya Sutskever, and Ruslan Salakhutdinov. 2014. Dropout: A Simple Way to Prevent Neural Networks from Overfitting. J. Mach. Learn. Res., 15(1):1929_ 1958.

Kristina Toutanova, Dan Klein, Christopher D. Manning, and Yoram Singer. 2003. Feature-rich Part-ofspeech Tagging with a Cyclic Dependency Network. In Proceedings of the 2003 Conference of the North American Chapter of the Association for Computational Linguistics on Human Language Technology - Volume 1, NAACL 2003, pages 173-180, Stroudsburg, PA, USA. Association for Computational Linguistics.

Yonghui Wu, Mike Schuster, Zhifeng Chen, Quoc V. Le, Mohammad Norouzi, Wolfgang Macherey, Maxim Krikun, Yuan Cao, Qin Gao, Klaus Macherey, Jeff Klingner, Apurva Shah, Melvin Johnson, Xiaobing Liu, Lukasz Kaiser, Stephan Gouws, Yoshikiyo Kato, Taku Kudo, Hideto Kazawa, Keith Stevens, George Kurian, Nishant Patil, Wei Wang, Cliff Young, Jason Smith, Jason Riesa, Alex Rudnick, Oriol Vinyals, Greg Corrado, Macduff Hughes, and Jeffrey Dean. 2016. Google's Neural Machine Translation System: Bridging the Gap between Human and Machine Translation. CoRR, abs/1609.08144.

Matthew D. Zeiler. 2012. ADADELTA: an adaptive learning rate method. CoRR, abs/1212.5701. 